\title{
SXRD measurements of residual stresses in sintered diamond-cobalt composites
}

\author{
H.-A. Crostack ${ }^{1, a}$, U. Selvadurai-Laß1 ${ }^{1, b}$, G. Fischer ${ }^{1, c}$, A. Rothkirch ${ }^{2, d}$ \\ ${ }^{1}$ Chair of Quality Management, University of Dortmund, Germany \\ Leonhard-Euler-Str. 5, 44227 Dortmund, Germany \\ ${ }^{2}$ HASYLAB at DESY, Noltkestr. 85, 22603 Hamburg, Germany
}

asekretariat@lqw.mb.un-dortmund.de, ${ }^{b}$ ursula.selvadurai.lassl@lqw.mb.uni-dortmund.de, cgottfried.fischer@lqw.mb.uni-dortmund.de, ${ }^{d}$ andre.rothkirch@desy.de

Keywords: cobalt-diamond composite, residual stresses, local measurements, synchrotron radiation

\begin{abstract}
Diamond-cobalt composites are used for cutting tools. Residual stress after manufacture can reduce the lifetime of such composite cutting tools and, hence, the stress state needs to be well understood. Within this study, stress measurements on a cobalt diamond composite were made by SXRD to deduce stress states in the cobalt matrix using the (222) reflection. The application of different apertures allowed the investigation of stress in small areas of cobalt surrounding a diamond and in areas at different distances to a diamond. In the areas adjacent to the diamond increased residual stresses were found in the cobalt matrix. Furthermore, approximations for radial and tangential residual stresses have been derived which show to be different.
\end{abstract}

\section{Introduction}

Due to their good mechanical and physical properties, cobalt-diamond composites can be used as cutting tools for machining of mineral materials. Diamonds possess an extreme hardness, which is about three times higher than the hardness of tungsten carbide and four times higher than the one of alumina [1,2]. Cobalt exhibits a good bonding to diamond and a high wear resistance, which is important to prevent diamond break-out [3].

Failure of cutting tools made of polycrystalline diamond-layers and sintered on WC/Co-substrate is induced by breaking of individual diamonds and delamination at the diamond/substrate interface. These failure mechanisms are attributed to high external stress and superposed residual stresses build-up at the interface due to the mismatch of the thermal expansion coefficient between diamond and cobalt $[4,5]$. The same failure mechanisms may exist in cobalt-diamond composites. Thermally induced tensile stress in the cobalt matrix and compressive stress in the diamond particles are expected during sintering of metal matrix composites that are reinforced with diamond particles. Superposition of these stresses by external stresses may result in an early break-out of diamond particles, interface debonding or diamond fracture. Those damages can also occur during the manufacturing process. Hence, residual stresses influence the lifetime. Thus the knowledge of the residual stress state, especially in the bonding between cobalt and diamond, is important.

Work carried out on the residual stresses in cobalt-diamond composites and in pure cobalt, which was manufactured by vacuum sintering and additionally hot-isostatically pressing, was presented previously [6,7]. In contrast to the expected tensionfree state in the pure cobalt, compressive residual stresses were found. This was attributed to the inhomogeneous green density after compaction of the powder and the resultant different sintering rates between the inner and outer portions of the sample. This previous work also showed that the compressive stresses are lower in the matrix of composites than in pure cobalt material. This was attributed to the mismatch of thermal expansion coefficients (TEC) between cobalt and diamond. The incorporation of diamond should lead to a superposition of compressive residual stresses, which were also found in pure 
cobalt with residual stresses created by the mismatch of TEC [12]. To study this superposition, residual stress measurements were performed on small cobalt areas $\left(1\right.$ and $\left.2 \mathrm{~mm}^{2}\right)$ near diamonds by synchrotron radiation.

\section{Experiments}

The samples studied here were manufactured by vacuum sintering at 1100 and $1200^{\circ} \mathrm{C}$, respectively. They are of pellet shape and have a diameter of $12-15 \mathrm{~mm}$ and a thickness of 2-4 $\mathrm{mm}$. The particle size of cobalt powder was below $40 \mu \mathrm{m}$ whereas the size of diamond particles ranges from $300-450 \mu \mathrm{m}$.

Another cobalt-diamond composite was manufactured under industrial condition. This specimen has an extent of $20 \times 3 \times 0.2$ $\mathrm{mm}^{3}$ and consists of fine grained $(2 \mu \mathrm{m})$ cobalt and $10 \mathrm{vol} . \%$ diamonds.

To avoid the interaction of residual stress fields initiated in diamond regions special samples with six isolated diamonds located at the surface were sintered, additionally. These samples allow to investigate the influence of a single diamond on the stress state of the adjacent cobalt matrix by measurements on small irradiated matrix areas at different distances to a single diamond. One of these samples is shown in Fig. 1. The circle illustrates the investigated area when applying a $1 \mathrm{~mm} \varnothing$ mask with a diamond in the centre.

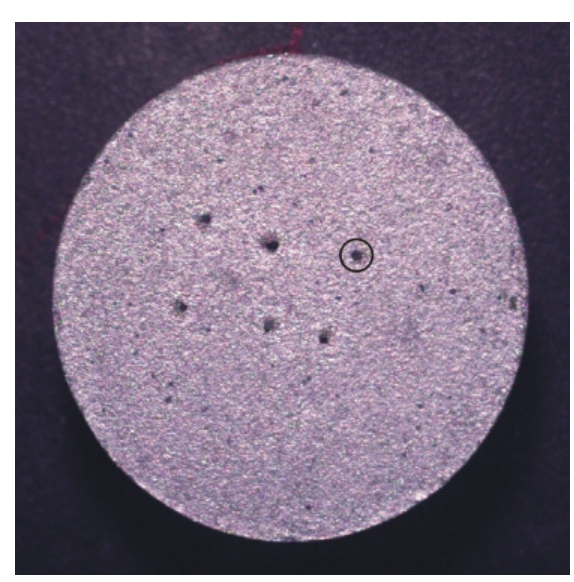

Fig. 1: Photo of a cobalt sample $(15 \mathrm{~mm} \varnothing)$ with six single diamonds.

The residual stresses in the cobalt matrix of these composites were measured by means of X-ray diffraction and the $d / \sin ^{2} \psi$-method [8] using synchrotron radiation at beamline G3 of HASYLAB at DESY, Hamburg, Germany.

Measurements have been carried out using a wavelength of $\lambda \approx 1.93 \AA$ (Fe- $\mathrm{K}_{\alpha}$ radiation) to analyse the (222)-reflection of cobalt $\left(2 \theta\right.$ angle of about $\left.142.2^{\circ}\right)$. Such high angles guarantee residual stress measurements with high precision and reduce the influence of texture [9]. In addition, measurements were made at a wavelength of $\lambda \approx$ of $1.66 \AA$ ( $\mathrm{Ni}-\mathrm{K}_{\alpha}$ radiation) to attain a higher penetration depth, using again the (222) reflection of cobalt for residual stress evaluation. Since the X-ray elastic constants of sintered cobalt are unknown, approximated values were calculated according to the method developed by Voigt [10] using a Young's modulus of $208 \mathrm{GPa}$ and a Poisson constant of 0.32 . The error of the stress values is given by the standard deviation from the slope of the straight line.

The residual stress measurements were carried out with different lateral resolutions and in different directions. In global measurement on industrial manufactured composites the residual stress was averaged across an area of about $6 \mathrm{~mm}^{2}$. In contrast, residual stress was analysed in areas sized $1 \mathrm{~mm}^{2}$ using the high brilliance and intensity of synchrotron radiation.

To irradiate such small areas, masks with different aperture size and shape were positioned on the sample surface at different distance to the diamonds. The masks with circular aperture (Fig. 2a) were fixed above the surface for sample rotation with a rotation axis lying in the diamond centre (Fig. 2a left, aperture $\varnothing 1 \mathrm{~mm}$ ) and in a matrix region $5 \mathrm{~mm}$ away from the diamond centre (Fig. 2a right, aperture $\varnothing 2 \mathrm{~mm})$. Due to rotation the residual stresses in all directions are averaged in this measurement ( $\sigma$-rotation). 


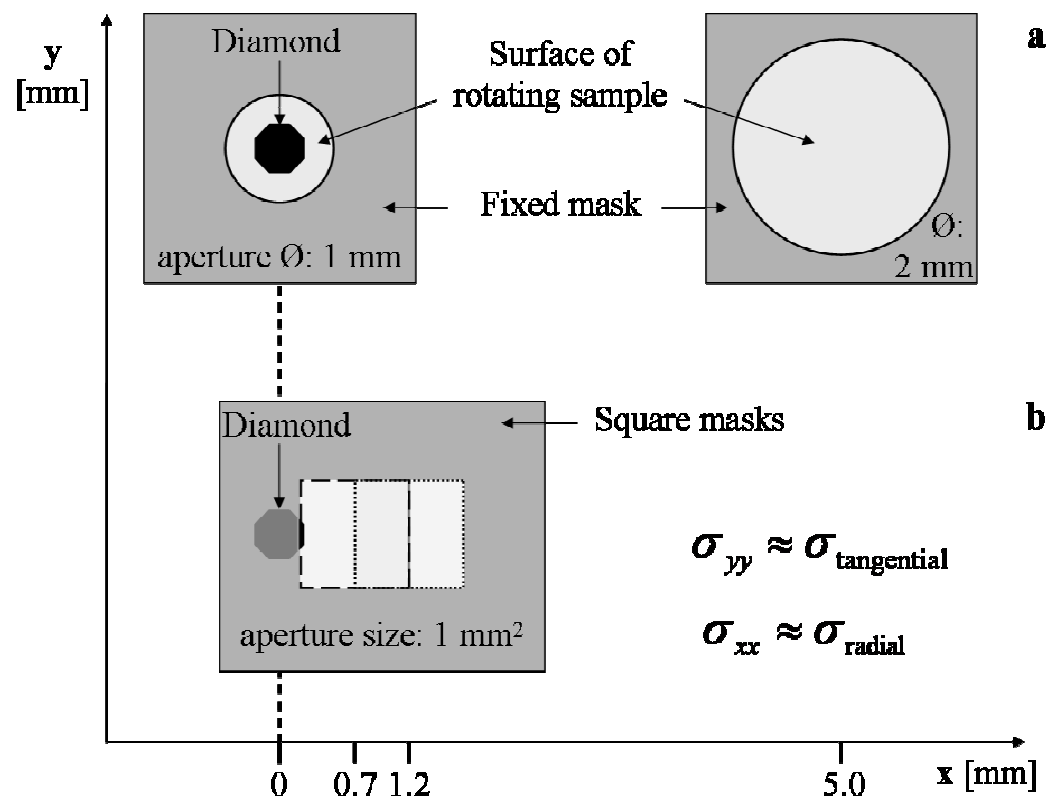

Fig. 2: Positions of masks and directions for measuring residual stresses
The masks with square aperture (Fig.2b) were fixed on the specimen surface at two positions away from the diamond centre $(0.7$ and $1.2 \mathrm{~mm})$. In this case, the residual stresses in $\mathrm{x}$ - and in $\mathrm{y}$ direction $\left(\sigma_{\mathrm{xx}}, \sigma_{\mathrm{yy}}\right)$ were measured in $\psi$-mode [11].

Different portions of radial and tangential stress contribute to local values of $\sigma_{x x}$ and $\sigma_{y y}$ within the irradiated areas due to their large size (compared to the size of diamonds). Nevertheless, we assume the average values of $\sigma_{\mathrm{xx}}$ and $\sigma_{\mathrm{yy}}$ being adequate approximations for the radial and tangential stress, respectively.

\section{Results and discussion}

As presented in a previous work [6,7] global compressive stresses (diameter of measuring area $15 \mathrm{~mm})$ were found in pure cobalt $(-80 \pm 3$ $\mathrm{MPa}$ ). These global compressive stresses were reduced to $-14 \pm 4 \mathrm{MPa}$ in the cobalt matrix of the composite by incorporating diamonds.

New global residual stress measurements carried out up to a penetration depth of $18 \mu \mathrm{m}$ on an industrial manufactured composite verify the existence of compressive stresses in the cobalt matrix of composites (Fig. 3). In this rectangular sample, the stresses were found to be dependent on direction and independent on depth. The stress component $\sigma_{\mathrm{xx}}$ (parallel to the longitudinal axis) is larger than the transverse component $\sigma_{\mathrm{yy}}$ due to the inhomogeneous contraction of the sample.

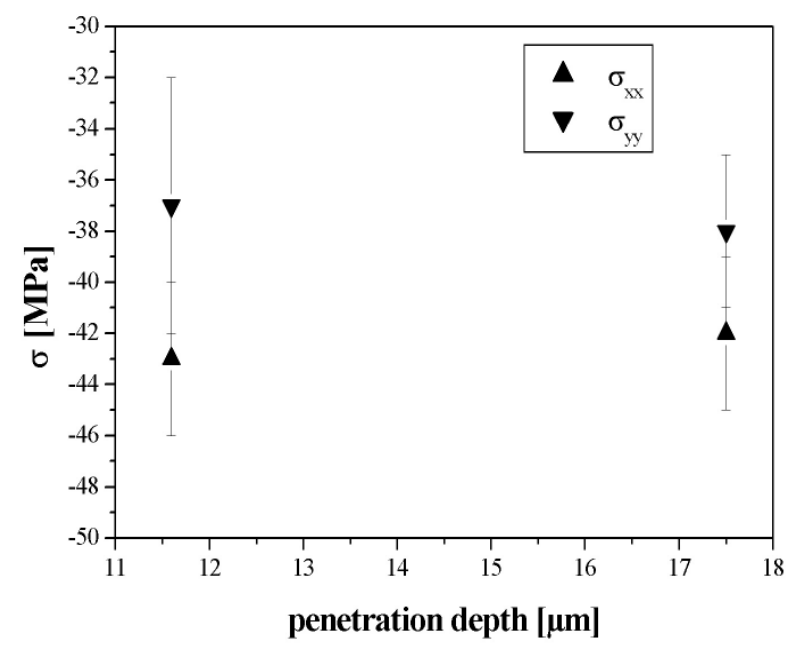

Fig. 3: Compressive residual stress components $\sigma_{\mathrm{xx}}$ and $\sigma_{\mathrm{yy}}$ in the industrially manufactured composite analysed with $\mathrm{Fe}-\mathrm{K}_{\alpha}$ and $\mathrm{Ni}-\mathrm{K}_{\alpha}$ radiation versus penetration depth

While the global measurements provide compressive residual stresses, measurements on a small area adjacent to a diamond executed on rotating samples result in tensile stresses at a distance of $0 \mathrm{~mm}$ (Fig. $4 \mathrm{a}+\mathrm{b}$ ) and $0.7 \mathrm{~mm}$ (Fig. $4 \mathrm{~b}$ ) to the diamond. With increasing distance these tensile stresses diminish and turns to compressive stresses at larger distances (between 1 and $5 \mathrm{~mm}$ ). The same measurements carried out in a distance of $5 \mathrm{~mm}$ to the diamond deliver compressive stresses (Fig. $4 \mathrm{a}+\mathrm{b}$ ). This shows that a tensile stress is induced in the matrix near to a diamond by the mismatch of TEC. The existence of tensile stress near the diamond explains the reduced compressive residual stress found in global measurement on the cobalt-matrix of sintered cobaltdiamond composites. 
The stresses $\sigma_{\mathrm{yy}}$ measured in y-direction adjacent to the diamond are positive (tensile stresses) for both sintering temperatures $\left(1100\right.$ and $1200^{\circ} \mathrm{C}$; Fig. 4). These stresses diminish as the distance to the diamond proceeds (Fig. 4a).

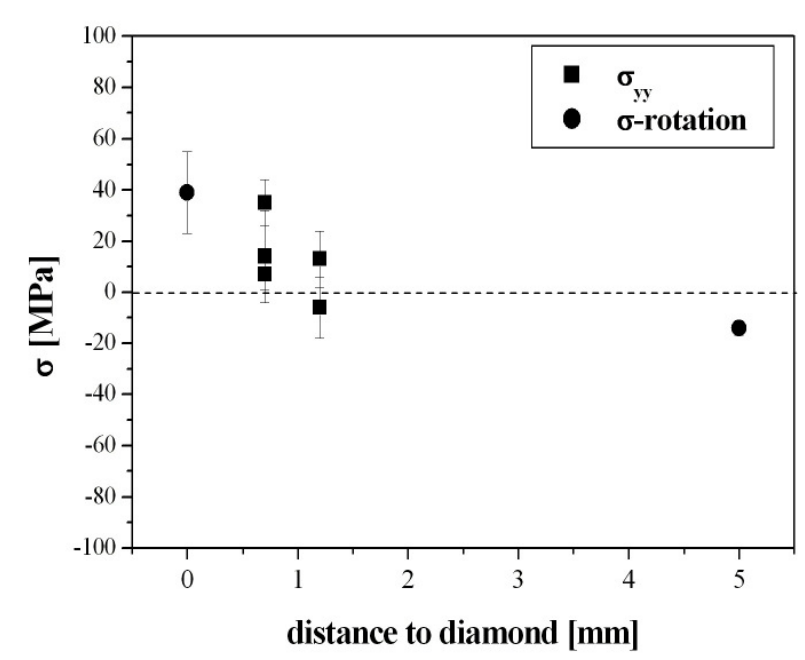

a)

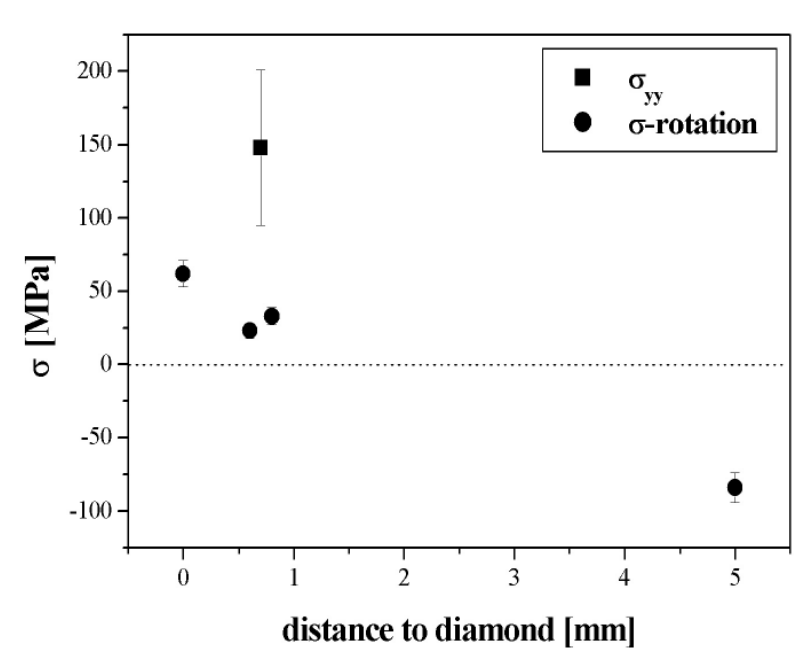

b)

Fig. 4: Residual stresses $\sigma_{\text {yy }}$ and $\sigma$-rotation as a function of distance relative to a diamond, a) sintering temperature $=1100{ }^{\circ} \mathrm{C}, \mathrm{b}$ ) sintering temperature $=1200{ }^{\circ} \mathrm{C}$

The comparison of Fig. $4 \mathrm{a}$ with $4 \mathrm{~b}$ indicates that the stress gradient increases with uprising sintering temperature. At $1200{ }^{\circ} \mathrm{C}$ sintering temperature the tensile stress near the diamond and the compressive stress at a larger distance from the diamond are much higher. The increased residual stress values are due to the effect of the mismatch of TEC. Tensile residual stress in y-direction can cause matrix cracks and the break-out of diamonds. Therefore, a sintering temperature of $1100{ }^{\circ} \mathrm{C}$ is recommended to increase the life time of such cutting tools.

In x-direction, a compressive stress was measured in the area adjacent to the diamond (centre position $x=0.7 \mathrm{~mm}$; Fig. 5). This stress component $\sigma_{\mathrm{xx}}$ turns into tensile stress at a distance of $1.2 \mathrm{~mm}$.

For comparison, first FE-simulations were performed assuming a hemispherical diamond ( $500 \mu \mathrm{m})$ incorporated in an elasto-plastic cobalt matrix (cylinder $\varnothing 6 \mathrm{~mm}, 1.5 \mathrm{~mm}$ thick). In Fig. 6 the curves of radial and tangential stresses at the model surface are plotted as a function of the distance to the centre of diamond. Corresponding to the stress $\sigma_{\mathrm{xx}}$ measured adjacent to the diamond, compressive radial stress were found in the simulation (Fig. 6a).

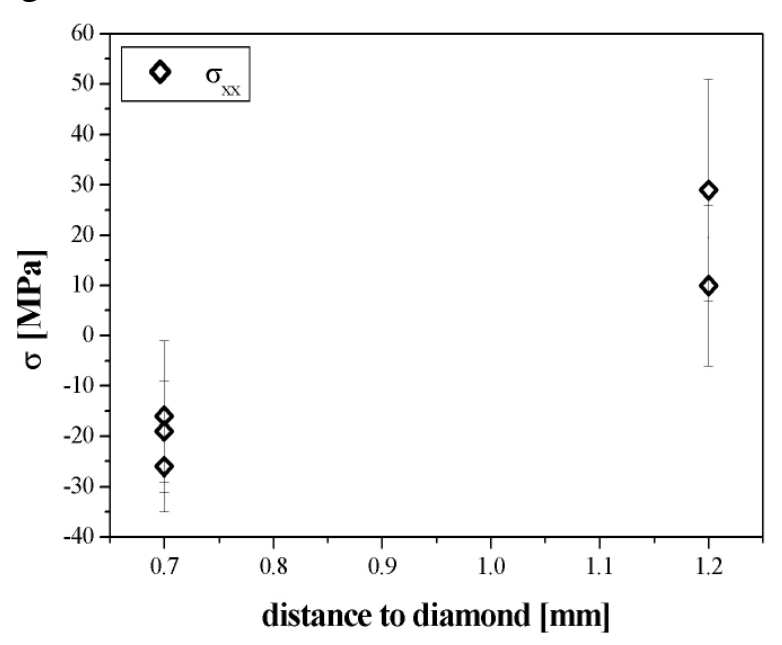

Fig. 5: Residual stress component $\sigma_{\mathrm{xx}}$ at two distances to the diamond.

As the distance increases the simulated radial stress turns into zero and not to a positive value measured at a distance of $1.2 \mathrm{~mm}$ to the diamond (Fig. 5). This is due to the fact that the influence of inhomogeneous sintering was not taken into consideration in the modelling. The curve of tangential stress (Fig. 6b) show a high tensile stress peak near the interface to the diamond $(\mathrm{x}=0.5$ $\mathrm{mm})$. This corresponds to the positive $\sigma_{\mathrm{yy}}$-values found in measurements adjacent to the diamond (Fig. 4). 


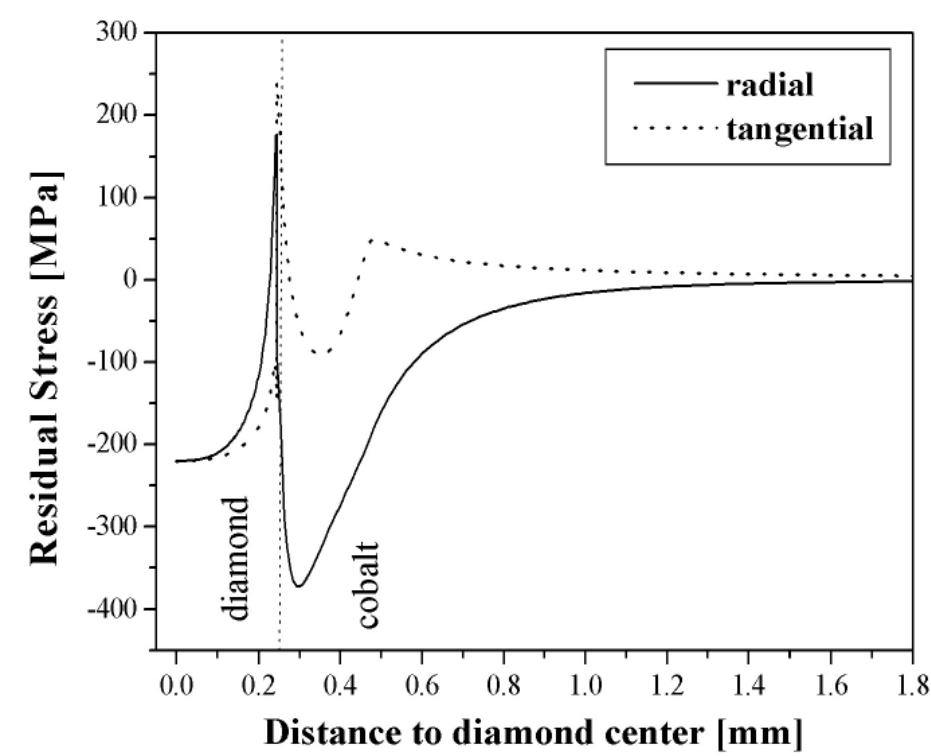

Fig. 6: FE-simulations of tangential and radial residual stresses in dependence of the distance to a diamond.

\section{Summary}

The results of X-ray diffraction measurements show that stresses in sintered cobalt-diamond composites are inhomogeneous. The global compressive residual stress in pure cobalt is reduced by incorporating diamond particles due to the mismatch of thermal expansion coefficients between diamond and cobalt. To verify this interpretation, residual stresses in small irradiated areas near a diamond were investigated. Using synchrotron radiation and applying small masks, the extent of an analysed area could be scaled down to $1 \mathrm{~mm}^{2}$. Different areas of the specimen were measured by positioning a mask at different positions onto or slightly above the sample surface leading to stress values as a function of distance to a diamond. Tensile stress was found in y-direction (assumed tangential) adjacent to diamond and compressive stress in x-direction (assumed radial). With increasing distance to the diamond the stress $\sigma_{\text {yy }}$ turns from tension to compression whereas the stress $\sigma_{\mathrm{xx}}$ turns from compression to tension. The sintering temperature has a significant effect on magnitude and gradient of the residual stress. In tangential direction, higher tensile stress was found for the specimen sintered at $1200{ }^{\circ} \mathrm{C}$ than for the one sintered at $1100{ }^{\circ} \mathrm{C}$. Because high stress can promote cracks and the break-out of diamonds, it might be concluded that the lower sintering temperature applied is recommended for the manufacturing process of cutting tools to optimise their lifetime. Additional residual stress measurements on diamonds and further FE-simulations are planned.

\section{Acknowledgements}

The authors would like to thank Dr. M. Gathen and C. Kronholz, Institute of Materials Engineering, University of Dortmund; and Dr. B. Wehner, Fa. Effgen for manufacturing diamondmetal matrix composites. This work was financially supported by the DFG ( $\mathrm{Cr} 4 / 110)$ which is gratefully acknowledged.

\section{References}

[1] W. Tillmann: Intern. Journal of Refractory Metals and Hard Materials 18 (2000), p. 301-306

[2] C. Marx: Druckschrift der Fa. Christensen Diamond Products GmbH, Celle (1967)

[3] M. del Villar, J. Echeberria, I. Iturriza, F. Castro: PM World Congress "Superhard/ Refractory Metals" (1998), p. 475-480 
[4] T.P. Lin, M. Hood, G.A. Cooper, X. Li: Wear 156 (1992), p. 135- 150

[5] T.P. Lin, M. Hood, G.A. Cooper, R.H. Smith: J. Am. Ceram. Soc. 77 (6), p. 1562- 68

[6] W. Tillmann, M. Klaassen, C. Kronholz, H.-A. Crostack, U. Selvadurai-Laßl: Euro PM 2005 Conference, Prag, 2.-5. October 2005, Vol. 1, (2005), p. 305-310

[7] H.-A. Crostack, U. Selvadurai-Laß1, W. Tillmann, M. Gathen, C. Krohnholz, T. Wroblewski, A. Rothkirch: Residual stresses in sintered diamond-cobalt composites". ECRS7, Berlin, 13.15. September 2006, Material Science Forum Vols. 524-525 (2006) pp. 787-792

[8] E. Macherauch, P. Müller: Z. f. angew. Physik 13 (1961), p. 305

[9] V. Hauk: Structural and Residual Stress Analysis by Nondestructive Methods, EvaluationApplication - Assessment. ELSEVIER 1997

[10]U. Wolfstieg: Psi-Goniometer. HTM 31 (1976), p. 19-22

[11]W. Voigt: Lehrbuch der Kristallphysik, Leipzig, B.G. Teuber Verlag (1928)

[12]H.-A. Crostack, U. Selvadurai-Laß1: Residual Stresses in Cobalt-Diamond Composites II. HASYLAB-Jahresbericht 2006 